\title{
Bases ecológicas para implementar un sistema de uso silvopastoral en la región patagónica de Aysén, Chile
}

\author{
L. Sánchez-Jardón 1,* \\ (1) Departamento de Conservación de Suelos y Agua y Manejo de Residuos Orgánicos, CEBAS-CSIC, Apdo. de correos 164, 30100 Espinardo, Murcia, \\ España.
}

* Autor de correspondencia: L. Sánchez-Jardón [laurasj@bio.ucm.es]

> Recibido el 27 de febrero de 2013, aceptado el 28 de mayo de 2013.

Sánchez-Jardón, L. (2013). Bases ecológicas para implementar un sistema de uso silvopastoral en la región patagónica de Aysén, Chile. Ecosistemas 22(2):119-121. Doi.: 10.7818/ECOS.2013.22-2.18

\section{Enfoque e interés del estudio}

La región de Aysén, en la Patagonia norte de Chile, representa una historia reciente de colonización (Ortega y Brüning 2004) en la que amplias extensiones de bosques caducifolios de lenga (Nothofagus pumilio [Poepp. et Endl.] Krasser) se transformaron, mediante grandes incendios forestales, en explotaciones ganaderas y fragmentos dispersos de estos bosques (Veblen et al. 1996). Actualmente existen dos sistemas de uso de los recursos naturales, el ganadero y el forestal, cuya gestión se ha desarrollado de manera independiente. La ganadería ha promovido la expansión de praderas mejoradas en detrimento de los bosques nativos, obviando el impacto positivo del árbol en el valor de producción y de conservación de los pastizales. El uso forestal, más reciente, se ha centrado en recursos exclusivamente forestales y ha favorecido la proliferación de plantaciones con coníferas exóticas. La Figura 1 muestra el paisaje característico de los valles ganaderos de la región. Esta investigación plantea generar los argumentos científicos para la implementación, por primera vez en la región, de un sistema integrado de gestión silvopastoral con lenga a partir de las sinergias resultantes de las interacciones ecológicas entre bosques y praderas, y que minimice el impacto negativo de la deforestación, como la pérdida de hábitat para especies nativas asociadas a los bosques originales o la intensa erosión de suelo.

El objetivo general de este trabajo es cuantificar y caracterizar el efecto de la cobertura arbolada de lenga sobre la estructura y productividad de los pastizales, en un contexto de uso ganadero y de conservación de valores naturales. Como hipótesis se propone que el arbolado mejora la producción para el ganado (producción de herbáceas y su calidad bromatológica) y el mantenimiento de valores naturales (diversidad de especies herbáceas y persistencia de las nativas) en la región. Para ello se examinan dos escenarios experimentales complementarios: 15 parcelas de dimensiones $20 \times 20 \mathrm{~m}$ a lo largo de un gradiente de cobertura arbolada, desde bosque denso a pradera abierta, y cuatro zonas de contacto entre bosque denso y pradera sin arbolado de $80 \times 30 \mathrm{~m}$ ubicadas en la finca experimental de INIA Tamel Aike ( $\left.45^{\circ} 58^{\prime} \mathrm{S}, 72^{\circ} 08^{\prime} \mathrm{W}\right)$. En éstas últimas, además, se instalaron exclusiones permanentes para evaluar el efecto del abandono ganadero (Fig. 2). Durante dos temporadas
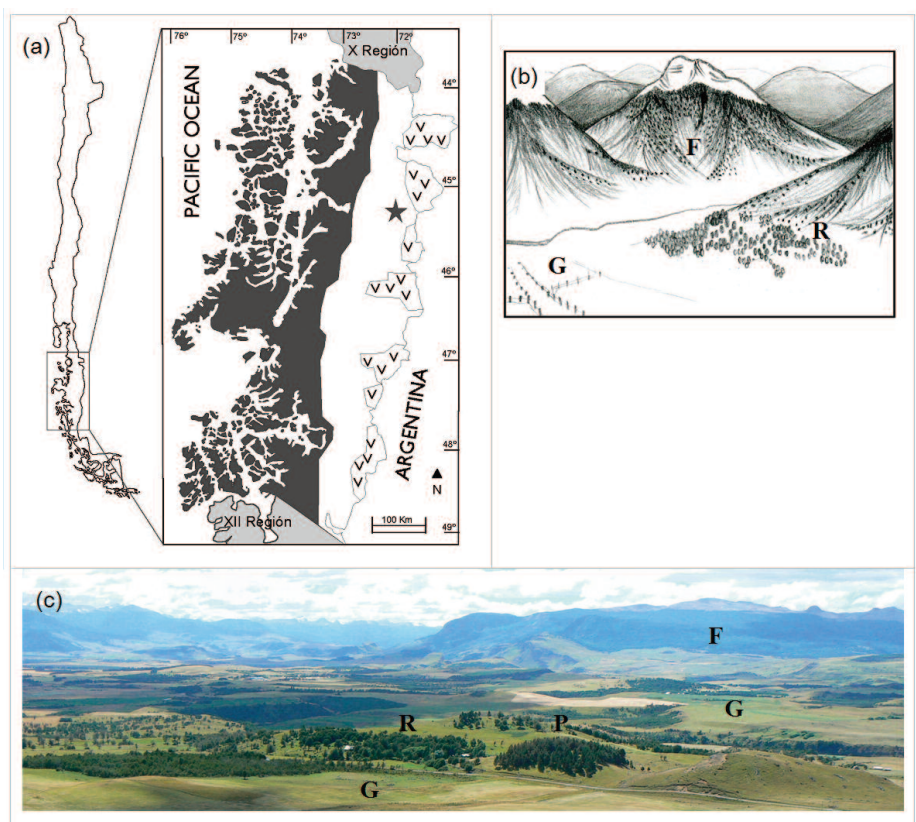

Figura 1. Mapa de situación geográfica de la región de Aysén en Chile (a) mostrando la distribución de la zona intermedia entre los bosques siempreverdes (área sombreada) y la estepa patagónica (v) dominan los bosques de lenga (área no sombreada); la estrella representa la ubicación geográfica de la ciudad de Coyhaique. Representación esquemática (b) y vista panorámica (c) del paisaje en un valle ganadero en la región en la actualidad. Después de los grandes incendios de principios del siglo $X X$, los bosques nativos se mantienen en las mayores altitudes $(F)$ y las praderas ganaderas en altitudes más bajas (G), donde también se pueden encontrar fragmentos remanentes de bosque nativo $(R)$ y plantaciones de coníferas exóticas $(P)$.

de crecimiento (octubre a abril de 2006-2007 y 2007-2008) se midieron variables ecológicas (estructura del dosel, cobertura de hojarasca, matorrales, troncos muertos y abundancia relativa de cada una de las especies registradas) y agronómicas (productividad primaria neta, contenido de proteínas, fibras y carbohidratos solubles) de los pastizales. También se registraron las plántulas de lenga, 


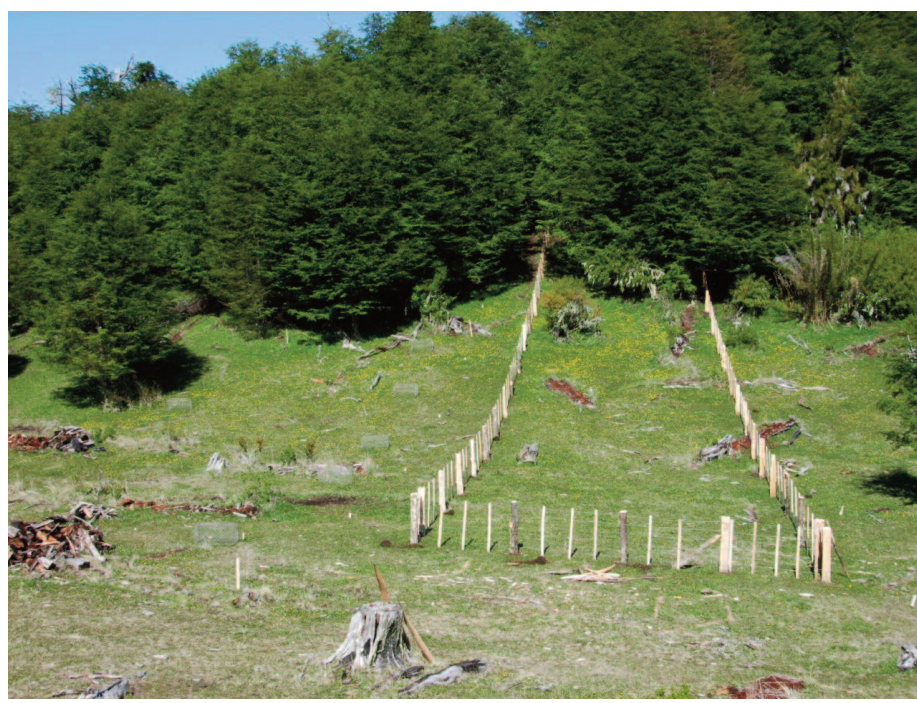

Figura 2. Fotografía de una de las cuatro parcelas de contacto entre bosque denso y pradera sin arbolado, con el correspondiente cerco de exclusión del ganado instalado al inicio de esta investigación, que simula el abandono ganadero.

para analizar la regeneración natural del bosque pastoreado con ganado. Los datos se analizaron mediante modelos lineales generales y análisis multivariante.

\section{Resultados y conclusiones generales}

Los resultados confirman el efecto positivo de ciertas coberturas de arbolado sobre las variables ecológicas y agronómicas de los pastizales. En general, la producción primaria neta herbácea (PPn) fue mayor en las praderas, aunque el $50 \%$ de los pastizales bajo arbolado presentaron valores de PPn superiores al promedio de las praderas. La variación de las variables de calidad en el gradiente de cobertura indicó que los pastizales bajo arbolado medio-denso presentan calidad intermedia, producto del compromiso entre su mayor valor nutritivo y su menor digestibilidad y palatabilidad respecto a praderas sin árboles (Sánchez-Jardón et al. 2010). Si bien en las praderas se registra mayor riqueza de herbáceas que en los bosques, este aumento es dependiente de la respuesta de las no nativas naturalizadas en la región; las nativas están restringidas a las zonas de arbolado más denso (Sánchez-Jardón et al., datos sin publicar). Estos resultados constituyen los principales argumentos científicos para avalar la implementación de sistemas silvopastorales con lenga en Aysén, como una alternativa de uso ganadero que mantiene la producción primaria y conserva parte del patrimonio natural asociado a los bosques nativos originales. La gestión ganadera deberá considerar que los bosques densos son necesarios para mantener una alta diversidad de especies nativas y la regeneración natural del bosque, ya que los árboles aislados o las áreas con arbolado poco denso no garantizan la persistencia de las plántulas de lenga, como tampoco de numerosas herbáceas nativas.

Las zonas arboladas que permiten alcanzar valores máximos de PPn son aquellas que permiten cierto paso de luz a su interior, idealmente entre $50-70 \%$ de radiación fotosintéticamente activa (PAR). Estos valores de radiación favorecen cierto consumo de hierba por parte del ganado, lo que desencadena un mecanismo de fertilización nitrogenada a través de sus excrementos que, a su vez, incrementa la producción herbácea (Fig. 3). El flujo de radiación puede lograrse con una cobertura medio densa de arbolado o bien mediante manchas de bosque denso pero con determinada configuración espacial; por ejemplo, aquellas de tamaño y formas que faciliten el paso de la radiación difusa a través de sus bordes en contacto con las praderas. La promoción de este tipo de manchas coexistiendo con otras de praderas abiertas podría considerarse un escenario deseable para un plan de gestión silvopastoral, preferible a un paisaje dominado por arbolado disperso de tipo sabanoide o por praderas sin arbolado.

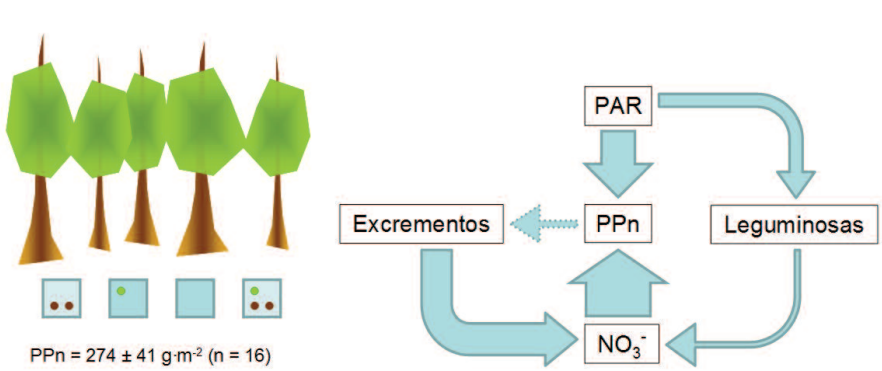

Figura 3. Esquema conceptual de los procesos que explican los altos valores de producción primaria herbácea (PPn) en bosques cuyo dosel permite la transmisión de 50-70\% de radiación PAR. Los puntos verdes indican la abundancia de leguminosas y los marrones la de excrementos y su tipo de distribución espacial, uniforme o concentrada. Las flechas en el diagrama de flujos representan las relaciones entre los diferentes compartimentos y su grosor indica el tamaño del compartimento donde termina la flecha.

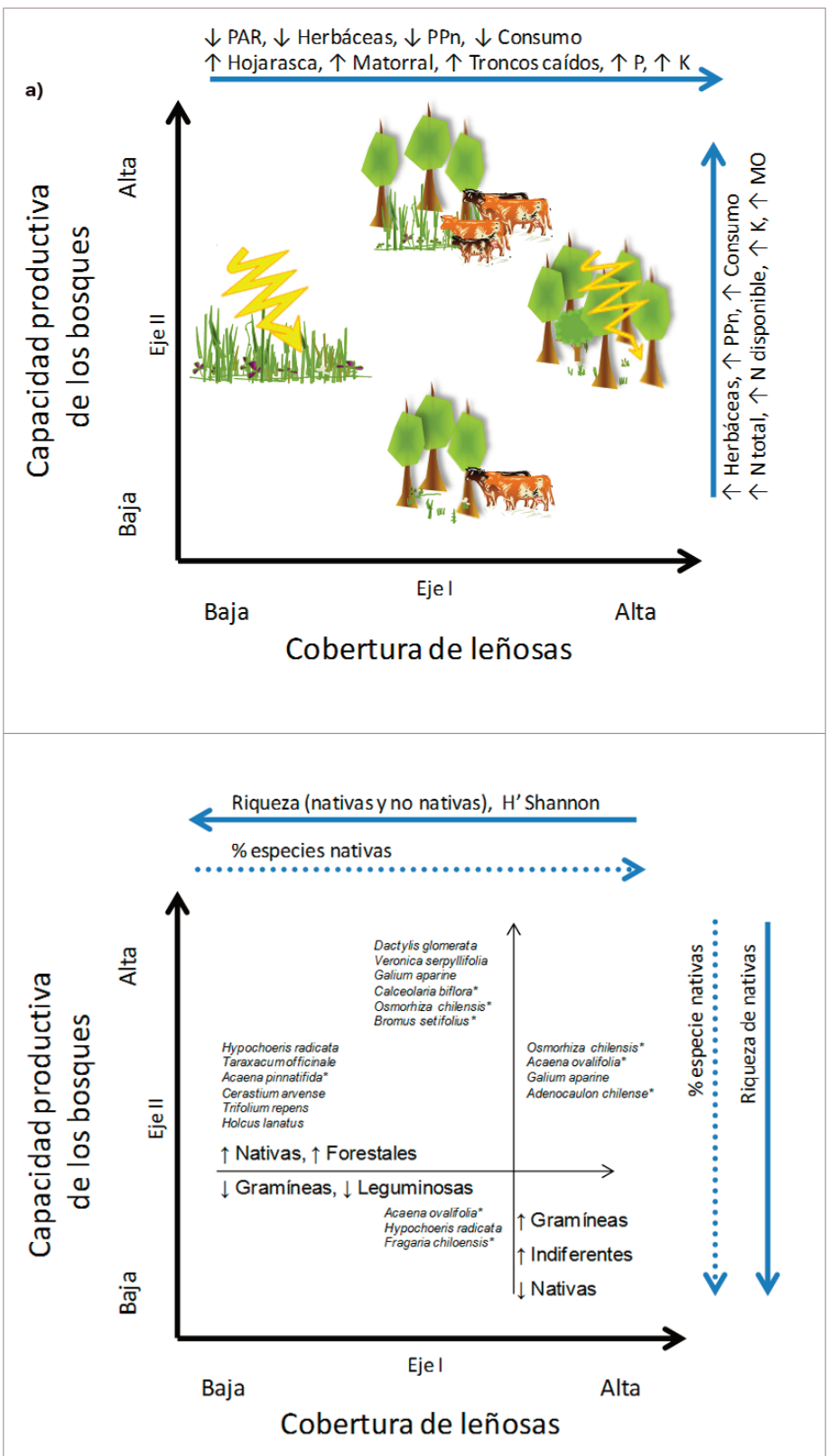

Figura 4. Esquema sintético de las principales tendencias de variación espacial de la estructura de los pastizales de bosques y praderas (a), y de su relación con las variables ecológicas y agronómicas analizadas (b). Los asteriscos $\left(^{*}\right)$ indican las species nativas según Marticorena y Quezada (1985). 
El efecto del arbolado, especialmente su papel como filtro de la luz, y el derivado del pastoreo por ganado son los dos factores principales que explican la variación espacial de la estructura (composición de especies, composición de atributos morfofuncionales y diversidad) de los pastizales (Fig. 4). Los matorrales en las praderas abiertas favorecieron la riqueza de herbáceas nativas y una composición florística más parecida a la típica de bosques, destacando su potencial protagonismo en la promoción de un paisaje silvopastoral, lo que cobra especial relevancia dada la lenta regeneración natural de la lenga. Finalmente, el abandono ganadero reciente altera la composición florística y la diversidad de los pastizales de forma diferente en bosques y en praderas, si bien en general favorece a las especies herbáceas nativas. Está previsto realizar análisis posteriores que consideren un tiempo de abandono más prolongado.

\section{Referencias}

Ortega, H., Brüning, A., 2004. Aisén. Panorama histórico y cultural de la XI Región. Ediciones LOM, Santigo, Chile. Disponible en: http://www.aisenpanorama.cl/libro.htm

Marticorena, C., Quezada, M., 1985. Catálogo de la flora vascular de Chile. Gayana Botanica 42:1-157.

Sánchez-Jardón, L., Acosta, B., del Pozo, A., Casado, M.A., Ovalle, C., Elizalde, H.F., Hepp, C., de Miguel, J.M., 2010. Grassland productivity and diversity on a tree cover gradient in Nothofagus pumilio in NW Patagonia. Agriculture, Ecosystems and Environment 137:213-218.

Veblen, T.T., Kitzberger, B., Rebertus, A., 1996. Perturbaciones y dinámica de regeneración en bosques andinos del Sur de Chile y Argentina. En: Armesto, J.J., Villagrán, C., Arroyo, M.K. (Eds.), Ecología de los bosques nativos de Chile, pp. 169-197. Editorial Universitaria, Santiago, Chile.

\section{LAURA SÁNCHEZ-JARDÓN}

\section{Bases ecológicas para implementar un sistema de uso silvopastoral en la región patagónica de Aysén, Chile}

\section{Tesis Doctoral}

Departamento de Ecología. Facultad de Biología, Universidad Complutense de Madrid

Fecha de lectura: 18/09/2012

Directores: José Manuel de Miguel Garcinuño y Alejandro del Pozo Lira

\section{Publicaciones resultantes de la tesis}

Sánchez-Jardón, L., Acosta, B., Elizalde, H.F., del Pozo, A., Casado, M.A., Ovalle, C., Hepp, C., De Miguel, J.M., 208. Grassland productivity and consumption under different tree cover in the Aysén region, Patagonia, Chile. Grassland Science in Europe 13;311-313.

Sánchez-Jardón, L., Acosta, B., Elizalde, H.F., del Pozo, A., Casado, M.A., Ovalle, C., Hepp, C., de Miguel, J.M., 2010. Floristic composi- tion and herbage quality changes with tree cover in NW Patagonia, Chile. Grassland Science in Europe 15;554-556.

Sánchez-Jardón, L., Acosta, B., del Pozo, A., Casado, M.A., Ovalle, C., Elizalde, H.F., Hepp, C., de Miguel, J.M., 2010. Grassland productivity and diversity on a tree cover gradient in Nothofagus pumilio in NW Patagonia. Agriculture, Ecosystems and Environment 137:213-218. 